Review paper

\title{
Update on latest advances in time-of-flight PET
}

\author{
Suleman Surti *, Joel S. Karp ${ }^{1}$ \\ Department of Radiology, University of Pennsylvania, Philadelphia, PA 19104, USA
}

\section{A R T I C L E I N F O}

\section{Keywords:}

Time-of-flight PET

Scintillator

SiPM

Coincidence timing resolution

\begin{abstract}
A B S T R A C T
This paper provides an update on time-of-flight (TOF) PET with a focus on latest hardware developments leading to current commercial PET/CT instruments. We describe advances in scintillator development, new photosensors and associated electronics readout, and detector designs for utilization in complete systems. Next, we introduce the latest commercial PET/CT scanners based on the aforementioned technologies, and discuss the detector design choices made that are relevant to differences in the system performance. Finally, we end with a discussion of the latest performance benchmarks for improved timing in PET detectors, challenges in scaling this performance to a complete system, and the outlook towards achieving a sub-50 ps coincidence timing resolution (CTR) in a PET detector.
\end{abstract}

\section{Introduction}

The first generation of TOF PET scanners developed in the $1980 \mathrm{~s}$ used $\mathrm{CsF}$ or $\mathrm{BaF}_{2}$ scintillators coupled to photomultiplier tubes (PMTs), achieving TOF resolution of 400-600 ps [1-6]. The low stopping power and low light output of these scintillators led to limited system sensitivity as well as poor spatial and energy resolution. The arrival of higher light output and dense lutetium-based (Lu-based) scintillators since the $1990 \mathrm{~s}$ [7] subsequently led to the development of a second generation of TOF PET scanners in the mid-2000 s with much higher system sensitivity and improved spatial resolution, while achieving TOF resolution in the 450-600 ps range [8-11].

The last five years have seen significant activity related to TOF PET hardware technology, especially in the area of photosensor development, leading to a widespread commercial introduction of silicon photomultiplier (SiPM) based (digital) whole-body TOF PET systems (third generation TOF PET systems) from all major manufacturers [12-16]. While the first SiPMs were developed in early 2000 s [17-21], it has taken several years of extensive research by various groups around the world, as well as new development by SiPM manufacturers, for these to be implemented in commercial PET systems. These new scanners not only provide much improved TOF resolution (as good as 214 ps [14]), but also have gains in sensitivity due to longer axial lengths and in some systems improved spatial resolution compared to the second generation of TOF PET systems. Most of these improvements were enabled by the development of compact SiPM photodetectors. In addition to providing improved intrinsic timing performance relative to the traditional PMTs, SiPMs also provide improved detector performance due to reduced or no signal multiplexing (average number of scintillation detectors per SiPM channel). In this aspect these designs are similar to the detectors originally designed for the first generation of TOF PET systems in the $1980 \mathrm{~s}$ that had no signal multiplexing (1-1 coupled), but were limited in spatial resolution due to the relatively large size of single PMTs, TOF resolution due to the scintillator and PMT characteristics, and sensitivity due to the scintillator density. Hence, the latest digital TOF PET system represent another generational step forward in the direction of improved PET image quality leading to ever shorter imaging times $(<10$ min for a whole-body scan) with potentially improved diagnostic performance.

This article provides a description of the PET hardware technology that has enabled the current generation of TOF PET, a design and performance review of the latest commercial whole-body PET/CT systems, and finally discusses future technological advancements in PET hardware. In particular the challenges in achieving 100 ps system TOF resolution in commercial PET with scintillation detectors, and the potential to go to sub-100 ps will also be discussed. Attaining sub-100 ps TOF resolution has significant implications for PET image generation and could open up new avenues for PET system design [22].

\footnotetext{
* Corresponding author at: Department of Radiology, Perelman School of Medicine at the University of Pennsylvania, 156B John Morgan Building, 3620 Hamilton Walk, Philadelphia, PA 19104, USA.

E-mail addresses: surti@pennmedicine.upenn.edu (S. Surti), joelkarp@pennmedicine.upenn.edu (J.S. Karp).

1 Address: Department of Radiology, Perelman School of Medicine at the University of Pennsylvania, 154 John Morgan Building, 3620 Hamilton Walk, Philadelphia, PA 19104, USA.
} 


\section{Advances in PET hardware technology}

System TOF resolution is directly related to the coincidence timing resolution (CTR) achieved in a PET scintillation detector. Detector CTR is determined by the scintillator (light yield, rise and decay times), photosensor (timing jitter and conversion efficiency), detector design (jitter due to variable depth-of-interaction or DOI, and multiple reflections in scintillator), and electronics (precision of timing measurement). Beyond the intrinsic timing performance of the detector (CTR), the system TOF resolution is also determined by calibrations and variability of detector performance. While Lu-based scintillators still provide the best combination of physical properties necessary for developing high performance TOF PET detectors, the last five years have shown that SiPMs not only provide improved intrinsic timing performance compared to PMTs but they also enable new detector designs that are capable of achieving an overall improved PET detector performance. Currently, several commercial systems using SiPMs have been developed, but the design and physical properties of SiPMs continue to improve leading to further advancements towards new and improved detector designs.

\subsection{Scintillators for TOF PET}

As mentioned earlier, Cerium-doped Lu-based scintillators, primarily LSO and LYSO but also LGSO [23] and LFS [24], currently provide the best combination of physical properties necessary for use in state-of-art commercial whole-body PET scanners. This is due to a combination of high stopping power with $\mu_{511}=0.78-0.88 \mathrm{~cm}^{-1}$ (necessary for system sensitivity), high light output of $31-34$ photons $/ \mathrm{keV}(\sim 17,000$ scintillation photons per $511 \mathrm{keV}$ photon that are necessary for good energy, spatial, and timing resolution), and short decay time $\left(\tau_{\mathrm{d}}\right)$ of $36-43 \mathrm{~ns}$ (necessary for low deadtime) [24-26]. All these characteristics make these scintillators a very good choice for use in fully-3D TOF PET scanners [27]. It has been shown [28,29] that CTR $\propto \sqrt{\tau_{r} \cdot \tau_{d} / N_{P h}}$ where $\tau_{\mathrm{r}}$ is the signal rise time and $\mathrm{N}_{\mathrm{Ph}}$ is the number of optical photons detected by the photodetector, which in turn is a function of light output, transmission probability through crystal, and detection efficiency of the photodetector. The high light output, short decay time, and fast rise time ( $\left.\tau_{\mathrm{r}},<1 \mathrm{~ns}\right)$ of Lu-based scintillators therefore lead to very good CTR, and hence the application for TOF PET imaging. With new doping methods there are potential benefits towards improved light output and shorter decay times - for LSO co-doped with Ca light output has been shown to increase to 39 photons $/ \mathrm{keV}$ with a decay time of $31 \mathrm{~ns}$ [30]. More recently, light output of up to 42 photons $/ \mathrm{keV}$ and shorter decay times ( $34 \mathrm{~ns}$ ) have been reported on for LYSO crystals co-doped with $\mathrm{Ca}$ and $\mathrm{Mg}$, and these improvements are being made at an industrial production level [31]. These improvements indicate a pathway towards improved timing performance with a new generation of Lubased scintillators as also shown with recent detector measurements (see below).

An alternate category of Cerium-doped rare earth garnet scintillators $\left(\mathrm{Gd}_{3} \mathrm{Al}_{2} \mathrm{Ga}_{3} \mathrm{O}_{12}\right.$ (GAGG) [32], $\mathrm{Gd}_{3}(\mathrm{Al}, \mathrm{Ga})_{5} \mathrm{O}_{12}$ (GGAG) [33], and (Gd, $\mathrm{Lu})_{3}(\mathrm{Al}, \mathrm{Ga})_{5} \mathrm{O}_{12}$ (GLuGAG)) [33]) have shown potential for favorable performance in fully-3D TOF PET scanners $\left(\mu_{511}=0.61-0.64 \mathrm{~cm}^{-1}\right.$, light output of 46-65 photons $/ \mathrm{keV}$, and decay time of 40-88 ns). Lu-based crystals require rare metals such as Iridium to make crucibles that can withstand $>2000^{\circ} \mathrm{C}$ temperatures for crystal growth, which adds significantly to crystal costs. The cubic structure of the garnet scintillators allows the use of ceramic techniques for faster crystal growth at much lower temperature and the ability to fabricate the scintillator in different shapes and sizes without cutting, thereby potentially leading to cost-effective manufacturing. However, as yet, no significant progress has been made in terms of developing large area PET detectors or prototype PET systems with these scintillators.

While BGO was the scintillator of choice for use in whole-body PET scanners starting from $1980 \mathrm{~s}$ through mid-2000 s, its low light output and slow scintillation signal were not good enough to perform TOF imaging. However, recent work has shown evidence that TOF imaging may be possible with BGO by detecting the prompt (almost instantaneous to within a few ps) Cerenkov photons that are emitted by the energetic electrons produced within the crystal due to absorption of the $511 \mathrm{keV}$ photons [34-36]. The Cerenkov photon emission yield is expected to be in the range of $10-30$ photons for $511 \mathrm{keV}$ photons, and is generally inversely proportional to the square of the Cerenkov photon wavelength [37]. For BGO this translates into $\sim 20$ Cerenkov photons per $511 \mathrm{keV}$ photon in the wavelength range of 305-750 nm [34]. After accounting for self-absorption in the crystal and the detection efficiency of the photosensor, the number of detected Cerenkov photons will be much lower. However, any TOF capability achieved via the Cerenkov mechanism in a BGO detector will provide additional advantages especially due to its much lower cost (about $1 / 3$ that of Lu-based scintillators).

\subsection{Photo-sensors for TOF PET}

The advent of SiPMs in the last 15-20 years has led to a significant shift in the design of PET detectors due to their high performance (high gain, low noise, and fast timing), compact design and ability to operate within a magnetic field [17-21]. A single SiPM device consists of several thousand reverse biased photo-diodes (or micro cells) operating near breakdown voltage on a common substrate. Each micro-cell operates in parallel with the other micro-cells and when scintillation photons reach the SiPM they initiate a Geiger avalanche in several micro-cells, leading to a current signal that is proportional to the number of micro-cells firing at any given time. As long as the number of incident scintillation photons is less than the number of micro-cells (otherwise, the SiPM saturates), the energy of the event will be proportional to the number of micro cells that fire, which is equivalent to integrating the analog signal. An integrated quenching mechanism using a resistor in series with each diode helps limit the device current and enables micro cell recovery. Device photodetection efficiency (PDE, probability for detecting an incident photon that is a product of device quantum efficiency, probability for triggering an avalanche, and the device fill-factor [21]) and single photon timing resolution (SPTR) are two major factors impacting its performance for fast timing applications. Any increase in PDE of the SiPM or increase in scintillator light output has a similar effect on CTR since both translate into a gain in the number of optical photons detected.

The very early SiPM devices were a few millimeters in size, had a low PDE of $10-15 \%$ at $420 \mathrm{~nm}$, but fairly good SPTR ( $<160 \mathrm{ps}$ fwhm) [38]. Despite a higher quantum efficiency of the individual microcells within these SiPM devices, the overall PDE was limited by the low fill factor of these devices. Technological developments in the last decade have led to much higher fill factors while limiting cross-talk between microcells, leading to the latest SiPM devices that provide PDE as high as 50-60\% at $420 \mathrm{~nm}$ [39] that is appropriate for very fast timing performance. Expansion of these individual SiPM devices into larger practical arrays is another technological milestone that has led to their use in modern commercial PET systems. SiPM arrays were originally fabricated from discrete SiPM devices connected via bond wires on a common printed circuit board, leading to large dead areas between individual SiPM channels and reduced PDE for the array. Recent developments using Through-Silicon Vias (TSV) technology to interconnect the individual SiPM channels in a larger array have significantly reduced the dead area due to classical wire bonding. A consequence of all these developments is the availability of large SiPM arrays with PDEs as high as 50-60\% at $420 \mathrm{~nm}$ [36] and SPTR of $<70 \mathrm{ps}$ [40]. The fabrication of the entire SiPM array on a common wafer (monolithic arrays) leads to minimal dead area between individual channels. However, replacing any defective channel in the array is impossible, leading to rejection of the full array and high fabrication costs. This limitation is not present in the 
discrete arrays where each channel can be individually chosen for good performance.

An alternate SiPM design integrates a dedicated 1-bit ADC for each micro cell on the substrate, which directly converts the micro cell state (fires or does not fire) into a digital signal [41-43]. An on-chip counter and timer lead to a fully-digital output of the incident photon energy and timing information leading to such devices being called digital SiPMs as opposed to the traditional SiPM also sometimes called analog SiPMs. A digital SiPM design has significant advantages over the traditional SiPM devices: it provides the ability to perform timing pickoff from the very first photon (and hence improved timing performance), it provides the ability to turn-off individual micro-cells, and most importantly it removes the need for dedicated back-end electronics in reading out large SiPM arrays. The current version of the digital SiPM technology provides all these advantages but there are some limitations in the design. A single time-to-digital converter (TDC) is currently used to obtain timing information from a single chip (4 channels) that leads to a timing skew based on which channel fires. Consequently SPTR values for this device are in the range of 150 ps [44]. Due to the integration of dedicated electronics on the chip, the fill factor for this device is also lower leading to a PDE of $\sim 25 \%$ at $420 \mathrm{~nm}$ [34]. Future technological developments such as use of a single TDC per channel are likely to improve the SPTR performance. However, improvements in PDE will require some major re-design in order to improve upon the device fill-detector. Development of 3D digital SiPMs with wafer-level integration of the SiPM and readout electronics [45] may eventually provide the maximum benefit of this approach. Such designs will allow utilizing all the intrinsic advantages of the traditional SiPMs (very high PDE, low SPTR) with the benefits of having a digital signal output. In particular, it may be possible to have a dedicated time-to-digital converter (TDC) for each microcell within a SiPM channel that will likely lead to a device with significantly improved intrinsic timing performance.

\subsection{TOF PET detector design and data acquisition electronics}

While the intrinsic properties of the scintillator and photosensor define the best CTR that can be achieved in a PET detector, it is also important to consider the need for spatial resolution and sensitivity in a full PET system. All commercial whole-body PET scanners until fairly recently have used Lu-based crystals that are 4-5 mm wide and 18-25 $\mathrm{mm}$ thick as the base detector. The crystal width has very recently been reduced to $<4 \mathrm{~mm}$ in commercial digital PET/CT available from at least two vendors $[14,15]$. In the past, significant signal multiplexing (number of crystals per photosensor channel) was used when using large PMTs to read out arrays of these narrow but long crystals. Detectors in the recent digital PET systems have greatly reduced or completely eliminated the signal multiplexing relative to the PMT-based detectors, but they still utilize similar narrow, long crystals. Multiple reflections of scintillation photons in a narrow, long crystal leads to a loss of some of these photons and also introduces a transit time dispersion, effects that degrade detector CTR [46]. Finally, the lack of depth-of-interaction (DOI) information also introduces a dispersion in time pickoff due to differences in speed for the scintillation and annihilation photons within the crystal that further degrades detector CTR [28]. With current pixelated detector designs where long crystals are read-out on back surface, there is no practical way to measure DOI. Hence, this leads to a degradation not only in the detector CTR, but it also degrades spatial resolution due to the well-known effect of parallax error. Despite these limitations, large pixelated crystal arrays still represent the detector of choice in all commercial whole-body PET systems primarily due to the simplicity of the design.

Data acquisition for PET systems using SiPM-based detectors can be challenging with a need to measure energy and timing from several tens of thousands of individual SiPM channels. Typically this requires the use of a dedicated TOF PET application specific integrated circuit (ASIC) chips with integrated amplifiers, non-delay line constant fraction discriminators (CFDs), and high precision TDCs [47-50]. Development of a dedicated ASIC is a costly and time consuming effort, but there are some commercial solutions available that are tailored for TOF PET applications. Considering the complexity of this task, the relative simplicity of the digital SiPM is apparent where all electronics are integrated within the device and only Field-Programmable Gate Arrays (FPGA) are needed to perform event processing.

\section{State-of-art in TOF PET scanners}

All major commercial vendors of PET/CT systems have transitioned their high-end product to a digital (SiPM-based) system and generally the axial field-of-view has increased to $>20 \mathrm{~cm}$ with one exception. The TOF resolution of these scanners varies in the range of 214-380 ps while the deadtime is very low compared to the traditional PMT based system due to minimal or no multiplexing [12-16]. Hence any differences in NEC performance, at least in the range of activities used for clinical imaging, are primarily determined by the system sensitivity. All of these systems also exclusively use Lu-based scintillators. In the following subsections we provide brief technical descriptions of these systems together with TOF resolution, spatial resolution, and sensitivity. The differences in spatial resolution and sensitivity are explained primarily by the choices made for crystal width and thickness as well as the system axial length. The differences in TOF resolution are dependent more on the choice of scintillator, SiPM array, and the detector design in terms of signal multiplexing and total light collection efficiency.

\subsection{GE Healthcare}

GE Healthcare has an axially scalable line of PET/CT under the GE Discovery MI series with axial lengths of 15 (3 rings), 20 (4 rings), and $25 \mathrm{~cm}$ (5 rings) (Fig. 1A) [13,51]. The basic crystal element (pixel) in these systems is a Lu-based scintillator with dimensions 3.95 (transverse) $\times 5.3$ (axial) $\times 25 \mathrm{~mm}^{3}$. A single optical block is composed of a 4 (transverse) $\times 3$ (axial) array of crystals. A 1 (transverse) $\times 3$ (axial) array of the optical blocks form a detector and 136 such detectors form a single ring of the scanner. Each detector is coupled to a non-contiguous 1 (transverse) $\times 3$ (axial) array of SiPM chips $(3 \times 2$ pixel array with pixel size of $4 \times 6 \mathrm{~mm}^{2}$ ) and an analog ASIC is used for signal processing and readout (Fig. 1B). Anger multiplexing is used for positioning with 2 crystals per SiPM pixel. A single scanner ring (15.0 cm axial length) is composed of 136 such detector blocks leading to a scanner ring diameter of $70 \mathrm{~cm}$. A closed-loop water cooling system is used to keep the SiPM arrays at a stable temperature of $19^{\circ} \mathrm{C}$. Signal multiplexing together with $<60 \%$ coverage of the crystal area $\left(\sim 754 \mathrm{~mm}^{2}\right)$ by the SiPMs $\left(\sim 432 \mathrm{~mm}^{2}\right)$ leads to a compromise in the system TOF resolution, which is measured to be $382 \mathrm{ps}$ [51]. Measured NEMA system sensitivity is $13.7 \mathrm{cps} / \mathrm{kBq}$ [13] and $20.8 \mathrm{cps} / \mathrm{kBq}$ [51] for the 4- and 5-ring systems, respectively, indicating the impact of scanner axial length. Reconstructed NEMA spatial resolution ( $\mathrm{fwhm}$ ) is $4.15 / 4.48 \mathrm{~mm}$ at $\mathrm{r}=1 \mathrm{~cm}$ and $6.22 / 6.1 \mathrm{~mm}$ at $\mathrm{r}=20 \mathrm{~cm}$ in the transverse/axial directions for the 4-ring system [13]. These numbers reflect the impact of Anger multiplexing and the use of thicker crystals that are longer in the axial direction compared to other commercial scanners; slightly poorer spatial resolution in the axial direction and also increased degradation at $r=20$ due to higher parallax error. For the 5-ring system reconstructed NEMA spatial resolution (fwhm) is $4.34 / 5.05 \mathrm{~mm}$ at $\mathrm{r}=1 \mathrm{~cm}$ and $6.20 / 6.56$ $\mathrm{mm}$ at $\mathrm{r}=20 \mathrm{~cm}$ in the transverse/axial directions [51]. A new Discovery MI Gen 2 has recently been announced that is similar to the current Discovery MI except for an axial length of $30 \mathrm{~cm}$, leading to even higher system sensitivity.

\subsection{United Imaging}

United Imaging offers two traditional PET/CT with a ring diameter of $76 \mathrm{~cm}$ that are identical in design except for the axial length: the uMI 


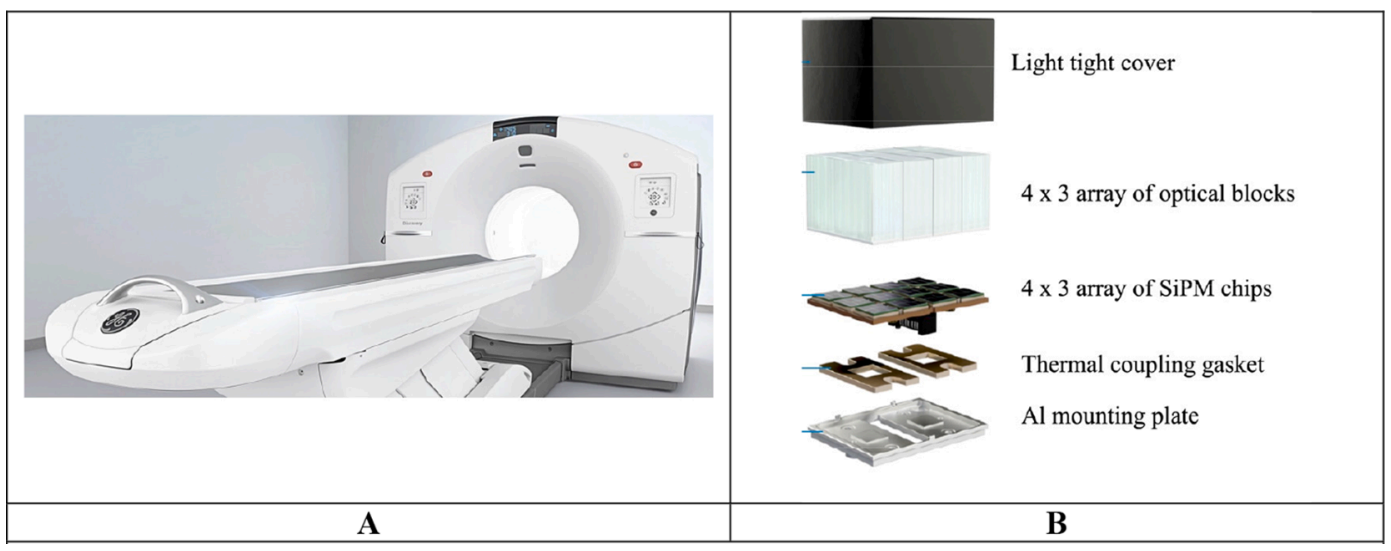

Fig. 1. (A) Picture of the GE Discovery MI PET/CT. (B) Picture of a $4 \times 3$ array of optical blocks coupled to a $4 \times 3$ array of SiPM chips [52]. Each optical block is a $4 \times$ 3 array of $3.95 \times 5.3 \times 25 \mathrm{~mm}^{3}$ Lu-based pixels and each SiPM chip is $3 \times 2$ pixel array with pixel size of $4 \times 6 \mathrm{~mm}^{2}$.

550 (24 cm axial length) [15] and uMI 780 (30 cm axial length) (Fig. 2A). The shared detector design is also common to their $194 \mathrm{~cm}$ total-body PET/CT - the UEXPLORER. The basic crystal element is a $2.76 \times 2.76 \times 18 \mathrm{~mm}^{3}$ LYSO pixel that is arranged in a 7 (transverse) $\times 6$ (axial) array block that is read-out by four non-contiguous $6 \times 6 \mathrm{~mm}^{2}$ single channel SiPMs in a light sharing block readout scheme (Fig. 2B). The multiplexing ratio is 10.5 crystals per SiPM channel. A 5 (transverse) $\times 14$ (axial) array of these blocks leads to a detector module that is $24.4 \mathrm{~cm}$ long in the axial direction. A scanner ring is composed of 22 of these modules with a ring diameter of $72.2 \mathrm{~cm}$. Signal readout electronics are based on traditional discrete circuits - amplification and leading-edge triggering for obtaining timing from the fast SiPM signal, and amplification and digitization (via analog-to-digital converter or $\mathrm{ADC}$ ) of the slow SiPM anode signal for calculating energy and position in an FPGA. System TOF resolution is reported as 372 ps at low activity levels [15], limited as in the GE systems primarily due to signal multiplexing and only $\sim 40 \%$ coverage of the crystal area $\left(\sim 341 \mathrm{~mm}^{2}\right)$ by the SiPMs $\left(\sim 144 \mathrm{~mm}^{2}\right)$. Measured NEMA system sensitivity is $10.2 \mathrm{cps} / \mathrm{kBq}$ for the uMI 550 with a reconstructed spatial resolution (fwhm) of 2.95/ $2.97 \mathrm{~mm}$ at $\mathrm{r}=1 \mathrm{~cm}$ and $4.11 / 3.13 \mathrm{~mm}$ at $\mathrm{r}=20 \mathrm{~cm}$ in the transverse/ axial directions [15].

\subsection{Siemens Healthineers}

Siemens Biograph Vision (Fig. 3A) represents the latest Siemens PET/CT using SiPM as the photosensor in the detector block [14]. The basic crystal element here is $3.2 \times 3.2 \times 20 \mathrm{~mm}^{3}$ LSO pixel. A $5 \times 5$ array of the individual pixels is coupled to a $4 \times 4$ channel SiPM array with full detector coverage to form a mini-block with multiplexing ratio of $\sim 1.56$ crystals per SiPM channel. A 4 (transverse) $\times 2$ (axial) array of these mini-blocks is packaged into a detector with signal readout performed with two custom-designed ASICs. A 2 (transverse) $\times 8$ (axial) array of these detector modules form a detector electronic assembly (DEA) with readout electronics (Fig. 3B). A scanner ring is composed of 19 such DEAs leading to a ring diameter of $78 \mathrm{~cm}$ and axial length of $26.1 \mathrm{~cm}$. The system is water-cooled at room temperature. Measured system TOF resolution is $214 \mathrm{ps}$ with system NEMA sensitivity of $16.4 \mathrm{cps} / \mathrm{kBq}$ [14]. The excellent TOF resolution of this system is due to low signal multiplexing ratio and $100 \%$ detector coverage by the SiPMs. Reconstructed NEMA spatial resolution ( $\mathrm{fwhm}$ ) is $3.55 / 3.50 \mathrm{~mm}$ at $\mathrm{r}=1 \mathrm{~cm}$ and 4.65/ $4.40 \mathrm{~mm}$ at $\mathrm{r}=20 \mathrm{~cm}$ in the transverse/axial directions [14].

\subsection{Philips Healthcare}

A pre-production version of the Philips Vereos (Fig. 4A) was originally developed and reported on as early as 2014 [54] but it was commercially available starting in 2017 [12]. This scanner uses the Philips Digital Photon Counting (PDPC) SiPM array (digital) as the photosensor. Each PDPC sensor is an 8x8 array of individual pixels 1-1 coupled to an $8 \times 8$ array of $3.86 \times 3.86 \times 19 \mathrm{~mm}^{3}$ LYSO pixels. The detector design for Vereos provides full light collection and is expected give the best timing performance for a given SiPM array. Fig. 4B shows a picture of the PDPC sensor placed next to an array of LYSO crystals and a complete detector. A $5 \times 4$ array of these detectors forms a detector

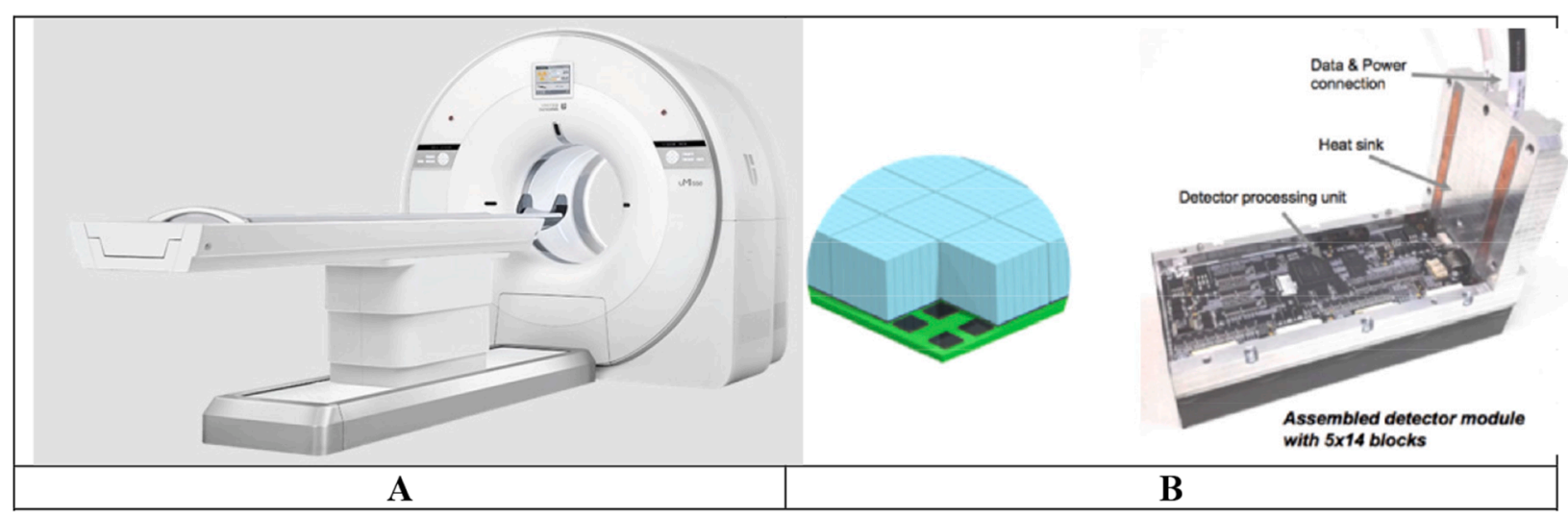

Fig. 2. (A) Picture of the United Imaging uMI 550 PET/CT. (B) Schematic (left) of an array of crystal blocks coupled to SiPMs (courtesy of Dr. Hongdi Li, United Imaging Healthcare America). Each crystal block is a $7 \times 6$ array of $2.76 \times 2.76 \times 18 \mathrm{~mm}^{3}$ LYSO pixels coupled to four non-contiguous $6 \times 6 \mathrm{~mm}^{2}$ single channel SiPMs in a light sharing block readout scheme. Picture of an assembled detector module (right) comprising of a $5 \times 14$ array of crystal blocks and associated electronics [53]. 


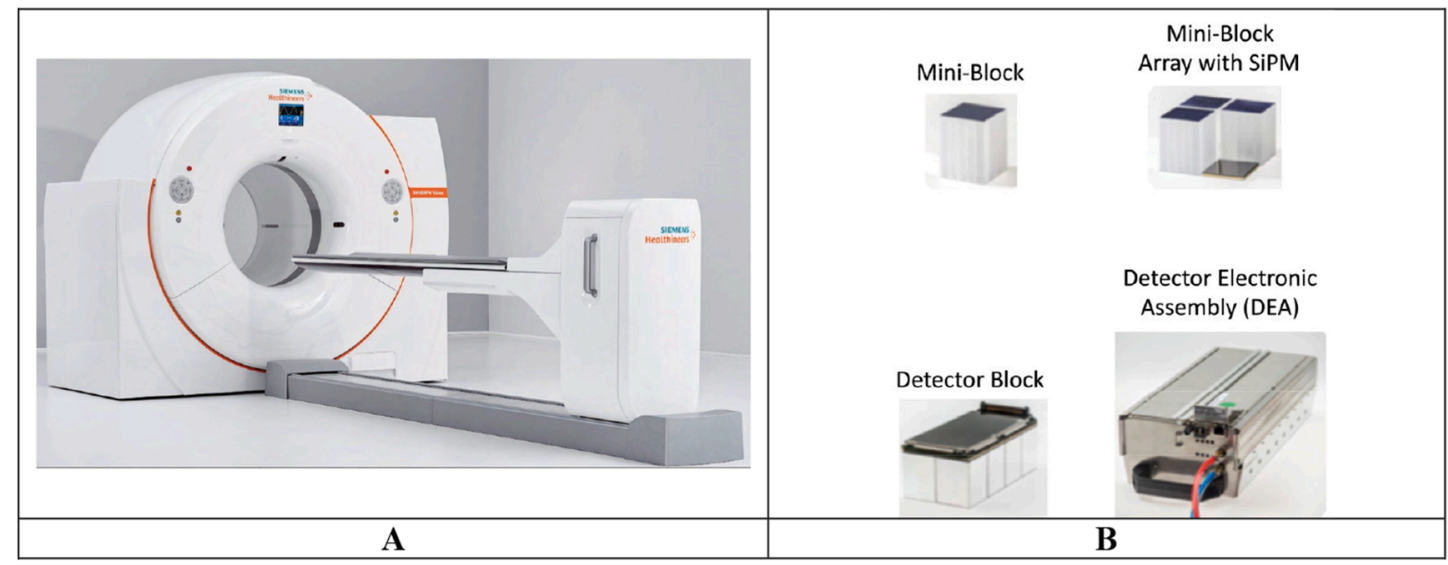

Fig. 3. (A) Picture of the Siemens Biograph Vision PET/CT. (B) Picture of a mini-block ( $5 \times 5$ array of $3.2 \times 3.2 \times 20 \mathrm{~mm}^{3}$ LSO pixels), a $2 \times 2$ array of mini-blocks coupled to a $2 \times 2$ array of SiPMs (each a $4 \times 4$ array of SiPM channels), a detector block composed of a $4 \times 2$ array of mini-blocks, and a detector electronic assembly (DEA) of comprising of $2 \times 4$ array of detectors and associated electronics. Pictures courtesy of Siemens Healthineers.

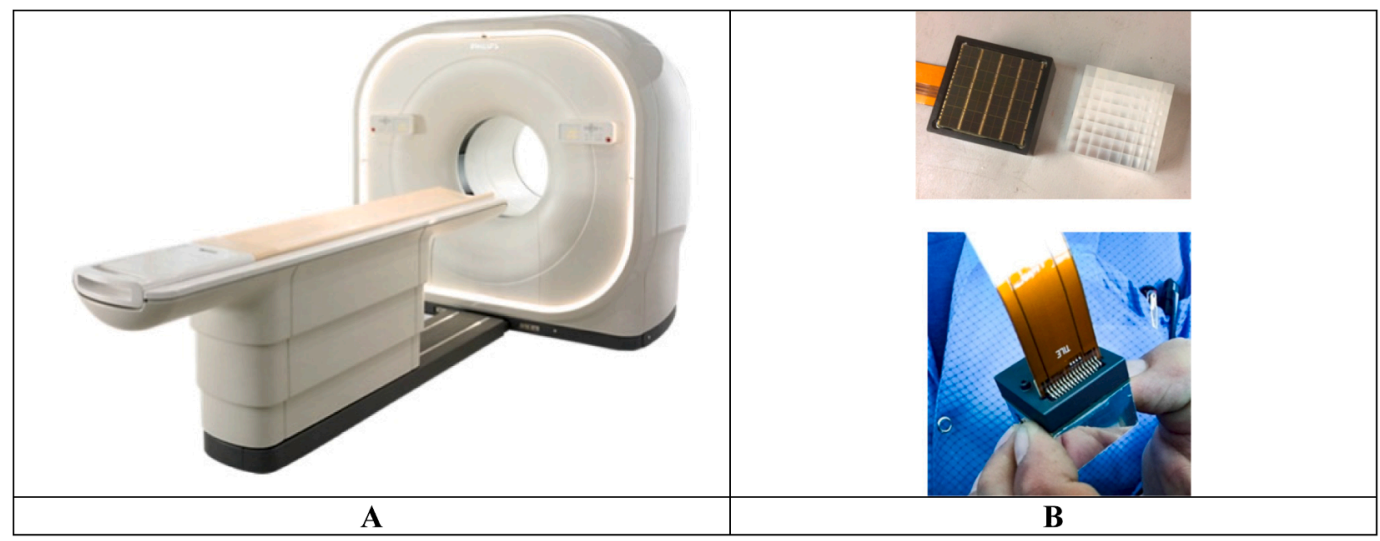

Fig. 4. (A) Picture of the Philips Vereos PET/CT. (B) Picture of an $8 \times 8$ array of $3.86 \times 3.86 \times 19 \mathrm{~mm}^{3}$ LYSO pixels next to an $8 \times 8$ array of the digital PDPC sensor (top) and the complete detector (bottom). Pictures courtesy of Philips Healthcare.

module and 18 such detector modules form the complete scanner ring with a diameter of $76.4 \mathrm{~cm}$ and axial length of $16.4 \mathrm{~cm}$. The system is also air-cooled and maintained at a stable temperature of $18^{\circ} \mathrm{C}$. Measured system TOF resolution according to NEMA NU2-2018 [55] methods is 332 ps [12]. Measured [12] system sensitivity of the Philips Vereos is $5.7 \mathrm{cps} / \mathrm{kBq}$ and the reconstructed spatial resolution (fwhm) is $4.01 / 3.96 \mathrm{~mm}$ at $\mathrm{r}=1 \mathrm{~cm}$ and $5.39 / 5.81 \mathrm{~mm}$ at $\mathrm{r}=20 \mathrm{~cm}$ in the transverse/axial direction according to NEMA NU2-2012 standards [56].

\subsection{Canon Medical Systems}

Cannon Medical Systems recently introduced their latest PET/CT also based on SiPM technology [16]. The new system, Cartesion Prime

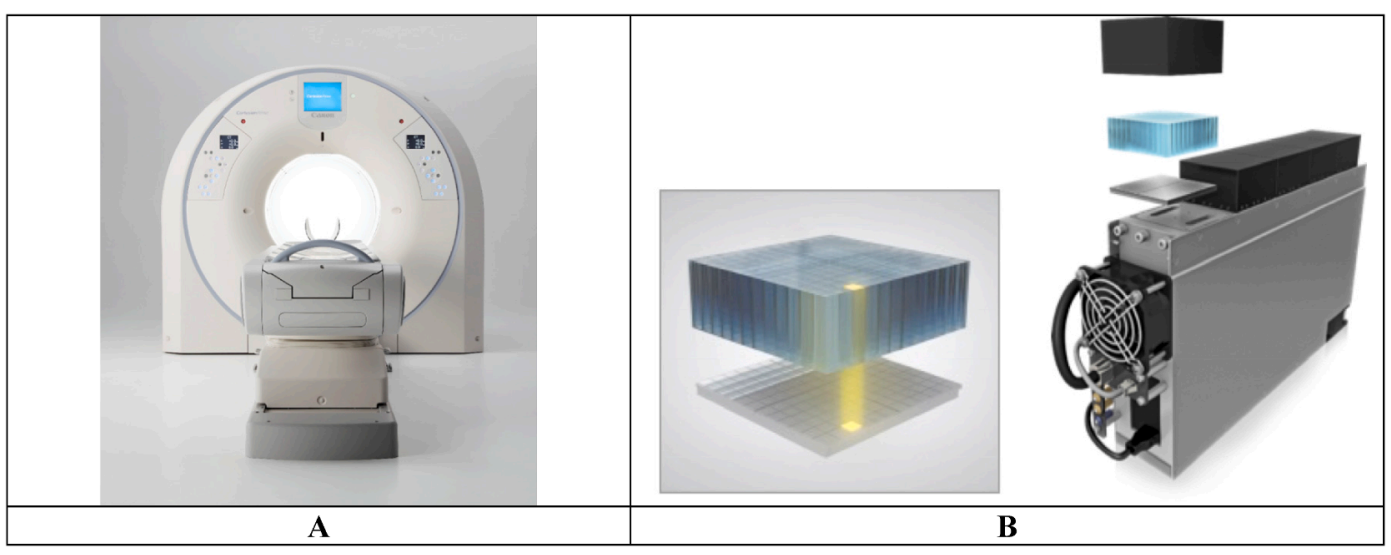

Fig. 5. (A) Picture of the Canon Medical Cartesion Prime PET/CT. (B) Schematic of a detector block (left) comprising a $12 \times 12$ array of $4.1 \times 4.1 \times 20 \mathrm{~mm}^{3}$ LYSO pixels 1-1 coupled to a $12 \times 12$ array array of SiPMs, and a detector module (right) with a $1 \times 5$ array of the detector blocks with associated electronics and an aircooling system. Pictures courtesy of Drs. Jeffrey Kolthammer and Maria Iatrou, Canon Medical Systems, USA. 
(Fig. 5A), uses $4.1 \times 4.1 \times 20 \mathrm{~mm}^{3}$ LYSO pixels. Each detector block is an array of $12 \times 12$ pixels $1-1$ coupled to a $12 \times 12$ array of SiPMs with complete (100\%) detector coverage (Fig. 5B). A 1 (transverse) $\times 5$ (axial) array of these blocks forms a single detector module that is aircooled to maintain stable room temperature. The scanner ring dimeter is $78 \mathrm{~cm}$ with axial length of $27 \mathrm{~cm}$. System TOF resolution is measured to be 258 ps with NEMA sensitivity of $13.5 \mathrm{cps} / \mathrm{kBq}$ [16].

\section{Performance benchmarks for CTR in SiPM-based PET scintillation detectors and challenges in scaling to a complete PET system}

\subsection{Lu-based detectors}

Measurements using single Lu-based crystals coupled to a single FBK NUV HD SiPM (SPTR of $68 \mathrm{ps} \mathrm{[40]} \mathrm{and} \mathrm{PDE} \mathrm{of} 60 \%$ at $410 \mathrm{~nm}$ [36]) have achieved a CTR of $58-80 \mathrm{ps}$, but this is achieved with very thin $(\sim 2 \mathrm{~mm}$ thick) crystals that do not provide sufficient stopping power necessary for PET $[57,58]$. With thicker $(\sim 20 \mathrm{~mm})$ crystals that is typical for PET scanners, CTR values in the range of 98-122 ps have been measured $[57,58]$. These bench-top measurements utilizing waveform sampling on high-end digital oscilloscopes indicate that CTR in the range of $100-150$ ps is achievable. However, expanding such a signal readout scheme to a full PET systems can be extremely challenging technically and also expensive. Recent work [59] with an array of $4 \times 4 \times 12 \mathrm{~mm}^{3}$ LYSO crystals 1-1 coupled to a KETEK SiPM array (similar PDE and SPTR as the FBK NUV HD device) and read out via PETsys TOFPET2 ASIC showed a CTR of 216 ps, matching the TOF resolution of the Siemens Vision scanner [14] (the current benchmark for commercial/ scalable designs), but significantly worse than the single channel benchtop measurements with specialized electronics. While these traditional, analog SiPM arrays have achieved very high intrinsic performance, a major limitation in using these devices in a system that achieves CTR performance in the range of 100-150 ps comes from the need to develop scalable electronics. A dedicated ASIC that takes full advantage of the intrinsic performance of these devices is a necessary area of development. Digital SiPMs with integrated electronics provide fast timing capability while also showing direct scalability from benchtop measurements to a full system $[12,41]$. Timing pickoff can be determined from the first photon trigger signal to achieve the best timing performance. However, this setting is also sensitive to the dark count rate of the device and will lead to increased deadtime. Hence, the commercial implementation of the digital SiPM in the Philips Vereos achieves system TOF resolution of 332 ps by performing timing pickoff when at least two photons are detected in the device, with low deadtime at a temperature at $18^{\circ} \mathrm{C}$. In contrast, the same digital SiPMs are used in the PennPET Explorer research scanner [60], which operates at $5^{\circ} \mathrm{C}$ so that the first photon can be used for timing measurements to achieve a system TOF resolution of 250 ps with low deadtime. Overall, however, the low PDE of these devices $(\sim 25 \%$ at $420 \mathrm{~nm})$ relative to the analog SiPMs likely limits their current performance. Large gains in system CTR performance can be expected if the PDE of these devices is increased to values closer to those seen in the traditional SiPMs.

While achieving system CTR of 100-150 ps is likely achievable with development of scalable electronics for regular SiPMs or improved PDE for digital SiPMs, achieving CTR of $<100$ ps will also require a reevaluation of the detector design. In particular, DOI measurement is needed to reduce uncertainties in timing pickoff and the timing jitter due to multiple photon reflections in long, narrow scintillator pixels needs to be reduced. Over the years methods have been developed for measuring DOI in pixelated detectors (two sided readout [61], offset crystal layers [62], reflector design [63], etc.), but the primary focus of many for these designs has been towards small animal or dedicated organ-specific PET scanner development. Incorporating these methods robustly for several thousands of crystals present in a whole-body PET scanner, and without negatively impacting the intrinsic CTR, can be a challenge. Also, it has not yet been determined to be cost-effective for whole-body imaging studies that are primarily ${ }^{18}$ F-FDG surveys for cancer diagnostics, and where point spread function (PSF) modeling methods can be effective in image reconstruction. Finally, reducing the effect of multiple reflections of scintillation photons within a long narrow pixel may be difficult to overcome. Hence, alternate designs such as monolithic detectors with a wide cross-section provide a pathway where the DOI measurement is obtained together with significantly reduced photon reflections within the crystal.

Measurements with a $2 \mathrm{~cm}$ thick monolithic LYSO crystal coupled to a digital SiPM array have demonstrated a spatial resolution of $<1.5 \mathrm{~mm}$ (FWHM) and CTR of $<150$ ps, together with a DOI measurement [64]. Improved PDE of such a SiPM array could potentially lead to further gains in the CTR. Potential drawbacks of this design are the need for significant detector calibrations and complexity of position and timing estimation algorithms. Thin ( $\leq 10 \mathrm{~mm}$ thick) monolithic crystals have been practically implemented in commercial pre-clinical [65] and breast imaging systems [66], and efforts are also underway to extend these methods to thicker $(1.5-2 \mathrm{~cm})$ monolithic LYSO detectors to achieve very good spatial resolution and DOI measurement using arrays of traditional analog SiPMs [67]. However, increased light spread in a thick monolithic detector leads to a low signal-to-noise ratio (SNR) in the electronics signal from each SiPM channel and a time-walk, both of which degrade detector CTR [68]. The monolithic detector design using digital SiPM has the ability to trigger on the very first photon [64], providing an intrinsic advantage over detectors using analog SiPMs. Alternately, a multi-layered detector can be assembled with thin slices of large cross-section scintillator, each layer having independent read-out and representing separate DOI [69] (see Fig. 6). The complexity of detector calibrations is significantly reduced and the reduced light spread may be sufficient to achieve good CTR with analog SiPMs. Signal readout from the multiple SiPM arrays in a complete system may, however, be practically difficult. Hence, while achieving CTR of $<100$ ps may be possible, attention needs to be paid to the practicality and robustness of the detector design.

Finally, efforts are underway to reduce detector CTR to well below $50 \mathrm{ps}$, and approach values closer to $10 \mathrm{ps}$ [22]. While development of any new scintillator with significantly better intrinsic timing performance relative to Lu-based scintillators cannot be discounted, it is more likely that mechanisms other than scintillation are needed to achieve the improved CTR goal. One avenue is to use the very fast Cerenkov photons (few ps time scale that is an order of magnitude or more faster than the scintillation process in Lu-based scintillators) that are produced due to the passage of charged electrons produced by the annihilation photons within a scintillator. The number of Cerenkov photons produced per $511 \mathrm{keV}$ photon can be very small ( $\sim 9$ in LSO) with emission wavelength in the range of 305-750 nm. Hence, achieving good timing measurement with the Cerenkov photons that is better than the best measurements with scintillation photons will require SiPM that have increased PDE especially in the UV range and better SPTR than the best devices available today (PDE of the FBK NUV HD device is $45 \%$ at 300 $\mathrm{nm}$ [36] while the SPTR is $68 \mathrm{ps}$ [34]). The low Cerenkov photon yield will also make the CTR even more susceptible to the effects of unknown DOI and multiple reflections in long thick pixels. Hence, utilizing Cerenkov photons for achieving CTR $<50$ ps in a Lu-based PET detector will require not only new SiPM technology but also new detector design.

\subsection{BGO detectors}

Compared to Lu-based scintillators, scintillation emission in BGO does not have good timing properties necessary for TOF PET performance. However, BGO has a higher Cerenkov photon yield per $511 \mathrm{keV}$ photon $(\sim 20)$. For fast timing using Cerenkov photons in BGO, results with $20 \mathrm{~mm}$ thick BGO show a timing distribution with full-width at half-maximum (fwhm) of 330-560 ps $[34,36]$ but with very long tails (Lorentzian shape). The tails in the timing distribution arise due to the 


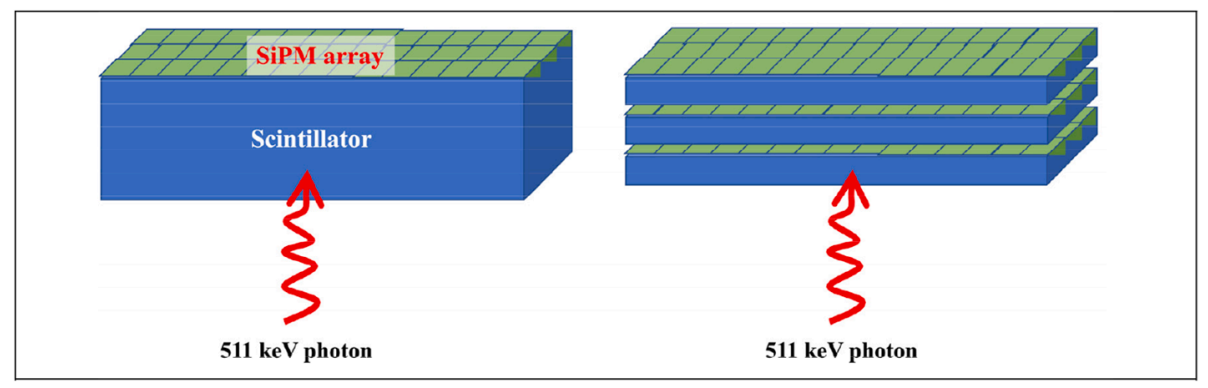

Fig. 6. Schematic of variations of a monolithic detector coupled to a SiPM array. At left is a thick monolithic detector and on the right are multiple layers of thin monolithic detectors.

nature of the BGO signal: a small but very fast signal due to Cerenkov photons together with a slower, but relatively much larger, scintillation signal. With dual-sided crystal readout, Kwon et. al. [70] have shown that the ambiguity in using Cerenkov or scintillation photons for timing can be reduced by using the earliest photon for timing on either end of the crystal. Results show that with this method the CTR fwhm for $20 \mathrm{~mm}$ thick BGO is improved from 463 ps to 399 ps at the fwhm level and from 1463 ps to 936 ps at the fwtm level [70]. Using high frequency (HF) electronics with fast oscilloscope readout, the CTR is further reduced to $331 \mathrm{ps}$ (fwhm) and $923 \mathrm{ps}$ (fwtm). Alternately, Kratochwil et. al. showed that fast waveform sampling (together with HF electronics) can be used to categorize each BGO signal into a range of fast to slow components based on rise time differences [71]. This can then be used to correct for time-walk effects and lead to CTR of 259 ps (fwhm) and 891 ps (fwtm) for $20 \mathrm{~mm}$ thick BGO [71]. While showing feasibility and setting benchmarks, use of two-sided readout in a full PET system with (laboratory) $\mathrm{HF}$ electronics, or using a very fast oscilloscope to categorize different rise times, do not easily lead to scalable, cost-effective solutions.

\section{Conclusion}

The re-introduction of TOF technology in PET in the mid-2000 s led to significant improvements in PET image quality even though the system TOF resolution was not improved over the first generation of TOF PET scanners. This was primarily due to the fact that these clinical systems, designed for whole-body FDG imaging, did not compromise sensitivity and spatial resolution. The last five years have seen significant progress made towards improving system CTR and currently five major commercial vendors have new digital PET/CT that achieve system TOF resolution in the range of 214-382 ps. The primary driver for these improvements has been the full embrace of SiPM photosensors that not only provide improved intrinsic timing performance compared to PMTs but also provide higher flexibility in detector design to reduce any deleterious effects arising from high multiplexing (number of scintillation detectors per photosensor channel). An additional advantage of these new detectors has been improved spatial resolution achieved in some of these clinical PET/CT with the use of smaller scintillation pixels.

Lu-based scintillators still provide the best combination of detector properties for use in PET, and benchtop measurements indicate that further improvements in CTR are likely. However, to achieve another factor of two or higher improvement in CTR will require advancements in cost-effective and scalable detector readout electronics for traditional SiPMs or gains in PDE of digital SiPMs, as well as potential reconsideration of the pixel-based scintillation detector designs. Research efforts are underway to achieve sub-50 ps CTR but these will require further advances in SiPM characteristics (e.g. improved SPTR) that are challenging to achieve, and also utilize non-scintillation mechanisms for better timing performance that may yet come at the expense of some other imaging characteristic of the PET detector.

\section{Declaration of Competing Interest}

The authors declare that they have no known competing financial interests or personal relationships that could have appeared to influence the work reported in this paper.

\section{Acknowledgments}

This work was supported by the National Institutes of Health grant Nos. R01-CA113941, R01-CA196528, R01-EB028764, and R21CA239177.

\section{References}

[1] Ter-Pogossian MM, Ficke DC, Hood Sr JT, Yamamoto M, Mullani NA. PETT VI: A positron emission tomograph utilizing cesium fluoride scintillation detectors. J Comput Assist Tomogr 1982;6:125-33.

[2] Ter-Pogossian M, Ficke D, Yamamoto M, JT H. Super PETT I: A Positron Emission Tomograph utilizing photon time-of-flight information. IEEE Trans Med Imag. 1982;M1-1:179-87.

[3] R. Gariod R. Allemand E. Cormoreche M. Laval Moszynski M. The "LETI" positron tomograph architecture and time-of-flight improvements. Proceedings of IEEE Workshop on Time-of-Flight Emission Tomography MO1982. Washington University St. Louis 2529.

[4] Wong WH, Mullani NA, Philippe EA, Hartz RK, Bristow D, Yerian K, et al. Performance characteristics of the University of Texas TOF PET-I Camera. J Nucl Med 1984;25:46-7.

[5] Lewellen TK, Bice AN, Harrison RL, Pencke MD, Link JM. Performance measurements of the SP3000/UW time-of-flight positron emission tomograph. IEEE Trans Nucl Sci 1988;35:665-9.

[6] Mazoyer B, Trebossen R, Schoukroun C, Verrey B, Syrota A, Vacher J, et al. Physical characteristics of TTV03, a new high spatial resolution time-of-tlight positron tomograph. IEEE Trans Nucl Sci 1990;37:778-82.

[7] Melcher C, Schweitzer JS. Cerium-doped lutetium oxyorthosilicate: a fast, efficient new scintillator. IEEE Trans Nucl Sci 1992;39:1161-6.

[8] Surti S, Kuhn A, Werner ME, Perkins AE, Kolthammer J, Karp JS. Performance of Philips Gemini TF PET/CT scanner with special consideration for its time-of-flight imaging capabilities. J Nucl Med 2007;48:471-80.

[9] Jakoby BW, Bercier Y, Conti M, Casey ME, Bendriem B, Townsend DW. Physical and clinical performance of the mCT time-of-flight PET/CT scanner. Phys Med Biol 2011;56:2375-89.

[10] Bettinardi V, Presotto L, Rapisarda E, Picchio M, Gianolli L, Gilardi MC. Physical Performance of the new hybrid PET/CT Discovery-690. Med Phys 2011;38: $5394-411$.

[11] Kolthammer JA, Su K-H, Grover G, Narayanan M, Jordan DW, Muzic RF, Performance evaluation of the Ingenuity TF PET/CT scanner with a focus on high count-rate conditions. Phys Med Biol 2014;59:3843-59.

[12] Zhang J, Maniawski P, Knopp MV. Performance evaluation of the next generation solid-state digital photon counting PET/CT system. EJNMMI Res 2018;8:97.

[13] Hsu DFC, Ilan E, Peterson WT, Uribe J, Lubberink M, Levin CS. Studies of a NextGeneration Silicon-Photomultiplier-Based Time-of-Flight PET/CT System. J Nucl Med 2017;58:1511-8.

[14] van Sluis J, de Jong J, Schaar J, Noordzij W, van Snick P, Dierckx R, et al. Performance characteristics of the digital Biograph Vision PET/CT system. J Nucl Med 2019;60:1031-6.

[15] Chen S, Hu P, Gu Y, Yu H, Shi H. Performance characteristics of the digital uMI550 PET/CT system according to the NEMA NU2-2018 standard. EJNMMI Phys 2020;7: 43.

[16] Li X, Qi W, Miyahara M, Kolthammer J. Performance Characterization of an SiPMbased Time-of-Flight Canon PET/CT Scanner. J Nucl Med 2020;61:14.

[17] Buzhan P, Dolgoshein B, Ilyin A, Kantserov V, Kaplin V, Karakash A, et al. An advanced study of silicon photomultiplier. ICFA Instrumentation Bull 2001;23:28. 
[18] Buzhan P, Dolgoshein B, Filatov L, Ilyin A, Kantzerov V, Kaplin V, et al. Silicon photomultiplier and its possible applications. NIM(A) 2003;504:48-52.

[19] Bisello D, Paccagnella A, Pantano D, Gotra Y, Malakhov N, Jejer V, et al. MetalResistive layer-Silicon (MRS) avalanche detectors with negative feedback. NIM(A) 1995;360:83-6.

[20] Golovin V, Saveliev V. Novel type of avalanche photodetector with Geiger mode operation. NIM(A) 2004;518:560-4.

[21] Renker D. Geiger-mode avalanche photodiodes, history, properties and problems. NIM(A) 2006;567:48-56.

[22] Lecoq P, Morel C, Prior J, Visvikis D, Gundacker S, Auffray E, et al. Roadmap toward the 10 ps time-of-flight PET challenge. Phys Med Biol 2020.

[23] Yamamoto S, Okumura S, Kato N, Yeom JY. Timing measurements of lutetium based scintillators combined with silicon photomultipliers for TOF-PET system. J Instrum 2015;10:T09002.

[24] http://www.zecotek.com/media/LFSWhitePaper.pdf.

[25] Spurrier MA, Szupryczynski P, Kan Y, Carey AA, Melcher CL. Effects of Ca2+ CoDoping on the Scintillation Properties of LSO:Ce. IEEE Trans Nucl Sci 2008;55: 1178-82.

[26] Pidol L, Kahn-Harari A, Viana B, Virey E, Ferrand B, Dorenbos P, et al. High efficiency of lutetium silicate scintillators, Ce-doped LPS, and LYSO crystals. IEEE Trans Nucl Sci 2004;51:1084-7.

[27] Muehllehner G, Karp JS, Surti S. Design considerations for PET scanners. Q J Nucl Med 2002;46:16-23.

[28] Moses WW, Derenzo SE. Prospects for time-of-flight PET using LSO scintillator. IEEE Trans Nucl Sci 1999;46:474-8.

[29] Moses WW. Time of flight in PET revisited. IEEE Trans Nucl Sci 2003;50:1325-30.

[30] Spurrier MA, Szupryczynski P, Yang K, Carey AA, Melcher CL. Effects of Ca2+ CoDoping on the Scintillation Properties of LSO:Ce. IEEE Trans Nucl Sci 2008;55: $1178-82$.

[31] Blahuta S, Yang K, Ouspenski V, Menge P, Perfromance engineering of LYSO single crystals. IEEE Nucelar Science Symposium \& Medical Imaging Conference. France: Strasbourg; 2016. p. 2016.

[32] Schneider FR, Shimazoe K, Somlai-Schweiger I, Ziegler SI. A PET detector prototype based on digital SiPMs and GAGG scintillators. Phys Med Biol 2015;60: 1667.

[33] Wang Y, Baldoni G, Rhodes WH, Brecher C, Shah A, Shirwadkar U, et al. Transparent garnet ceramic scintillators for gamma-ray detection. Hard X-Ray, Gamma-Ray, and Neutron Detector Physics XIV2012. p. 17-8.

[34] Brunner SE, Schaart DR. BGO as a hybrid scintillator / Cherenkov radiator for costeffective time-of-flight PET. Phys Med Biol 2017;62:4421-39.

[35] Brunner SE, Gruber L, Marton J, Suzuki K, Hirtl A. Studies on the Cherenkov Effect for Improved Time Resolution of TOF-PET. IEEE Trans Nucl Sci 2014;61:443-7.

[36] Kwon SI, Gola A, Ferri A, Piemonte C, Cherry SR. Bismuth germanate coupled to near ultraviolet silicon photomultipliers for time-of-flight PET. Phys Med Biol 2016;61:L38.

[37] Jelley JV. The Cerenkov Effect and its Applications. London: Pergamon; 1958.

[38] Collazuol G, Ambrosi G, Boscardin M, Corsi F, Dalla Betta GF, Del Guerra A, et al. Single photon timing resolution and detection efficiency of the IRST silicon photomultipliers. NIM(A) 2007;581:461-4.

[39] Piemonte C, Acerbi F, Ferri A, Gola A, Paternoster G, Regazzoni V, et al. Performance of NUV-HD Silicon Photomultiplier Technology. IEEE Trans Electron Dev 2016;63:1111-6.

[40] Cates JW, Gundacker S, Auffray E, Lecoq P, Levin CS. Improved single photon time resolution for analog SiPMs with front end readout that reduces influence of electronic noise. Phys Med Biol 2018;63:185022.

[41] T. Frach G. Prescher C. Degenhardt R. de Gruyter A. Schmitz R. Ballizany The digital silicon photomultiplier: Principle of operation and intrinsic detector performance FL2009. Orlando 19591965.

[42] C. Degenhardt G. Prescher T. Frach A. Thon R. de Gruyter A. Schmitz et al. The digital Silicon Photomultiplier; A novel sensor for the detection of scintillation light FL2009. Orlando 23832386.

[43] Schaart DR, Charbon E, Frach T, Schulz V. Advances in digital SiPMs and their application in biomedical imaging. NIM(A) 2016;809:31-52.

[44] Brunner SE, Gruber L, Hirtl A, Suzuki K, Marton J, Schaart DR. A comprehensive characterization of the time resolution of the Philips Digital Photon Counter. J Instrum 2016;11:P11004.

[45] Nolet F, Lemaire W, Dubois F, Roy N, Carrier S, Samson A, et al. A 256 Pixelated SPAD readout ASIC with in-Pixel TDC and embedded digital signal processing for uniformity and skew correction. NIM(A) 2020.

[46] Wiener RI, Surti S, Karp JS. DOI Determination by Rise Time Discrimination in Single-ended Readout for TOF PET Imaging. IEEE Trans Nucl Sci. 2013;60:147886.
[47] Rolo MD, Bugalho R, Gonçalves F, Mazza G, Rivetti A, Silva JC, et al. TOFPET ASIC for PET applications. J Instrum. 2013;8:C02050.

[48] Choong WS, Peng Q, Vu CQ, Turko BT, Moses WW. High-performance electronics for time-of-flight PET systems. J Instrum. 2013;8:T01006.

[49] Matsuda H, Kataoka J, Ikeda H, Kato T, Anbe T, Nakamura S, et al. Development of ultra-fast ASIC for future PET scanners using TOF-capable MPPC detectors. NIM(A) 2013;699:211-5.

[50] Sarasola I, Nemallapudi MV, Gundacker S, Sánchez D, Gascón D, Rato P, et al. A comparative study of the time performance between NINO and FlexToT ASICs. J Instrum. 2017.

[51] Pan T, Einstein SA, Kappadath SC, Grogg KS, Lois Gomez C, Alessio AM, et al, Performance evaluation of the 5-Ring GE Discovery MI PET/CT system using the national electrical manufacturers association NU 2-2012 Standard. Med Phys 2019;46:3025-33.

[52] Levin CS, Maramraju SH, Khalighi MM, Deller TW, Delso G, Jansen F. Design Features and Mutual Compatibility Studies of the Time-of-Flight PET Capable GE SIGNA PET/MR System. IEEE Trans Med Imag 2016;35:1907-14.

[53] Lv Y, Lv X, Liu W, Judenhofer MS, Zwingenberger A, Wisner E, et al. Mini EXPLORER II: a prototype high-sensitivity PET/CT scanner for companion animal whole body and human brain scanning. Phys Med Biol 2019;64:075004.

[54] Miller M, Griesmer J, Jordan D, Laurence T, Muzic R, Narayanan M, et al. Initial characterization of a prototype digital photon counting PET system. Society of Nuclear Medicine Annual Meeting Abstracts. 2014;55:658.

[55] NEMA. NEMA Standards Publication NU 2 2018. Performance Measurements of Positron Emission Tomographs. Rosslyn, VA: National Electrical Manufacturers Association; 2018.

[56] NEMA. NEMA Standards Publication NU 2 2012. Performance Measurements of Positron Emission Tomographs. Rosslyn, VA: National Electrical Manufacturers Association; 2012.

[57] Cates JW, Levin CS. Advances in coincidence time resolution for PET. Phys Med Biol. 2016;61:2255-64.

[58] Gundacker S, Turtos RM, Kratochwil N, Pots RH, Paganoni M, Lecoq P, et al. Experimental time resolution limits of modern SiPMs and TOF-PET detectors exploring di. Phys Med Biol. 2020;65:025001.

[59] Nadig V, Schug D, Weissler B, Schulz V. Evaluation of The PETsys TOFPET2 ASIC In Multi-Channel Coincidence Experiments. EJNMMI Phys 2020.

[60] Karp JS, Vishwanath V, Geagan M, Muehllehner G, Pantel A, Parma M, et al. PennPET Explorer: Design and Preliminary Performance of a Whole-body Imager. J Nucl Med 2020;61:136-43.

[61] Moses WW, Derenzo SE. Design Studies for a PET Detector Module Using a Pin Photodiode to Measure Depth of Interaction. IEEE Trans Nucl Sci 1994;41:1441-5.

[62] Robar JL, Thompson CJ, Murthy K, Clancy R, Bergman AM. Construction and calibration of detectors for high-resolution metabolic breast cancer imaging. NIM (A) 1997;392:402-6.

[63] Tsuda T, Murayama H, Kitamura K, Yamaya T, Yoshida E, Omura T, et al. A fourLayer depth of interaction detector block for small animal PET. IEEE Trans Nucl Sci. 2004;51:2537-42.

[64] Borghi G, Peet BJ, Tabacchini V, Schaart DR. A $32 \mathrm{~mm} \times 32 \mathrm{~mm} \times 22 \mathrm{~mm}$ monolithic LYSO: Ce detector with dual-sided digital photon counter readout for ultrahigh-performance TOF-PET and TOF-PET/MRI. Phys Med Biol 2016;61: 4929-49.

[65] Krishnamoorthy S, Blankemeyer E, Mollet P, Surti S, Van Holen R, Karp JS. Performance evaluation of the MOLECUBES $\beta$-CUBE-a high spatial resolution and high sensitivity small animal PET scanner utilizing monolithic LYSO scintillation detectors. Phys Med Biol 2018;63:155013.

[66] Moliner L, González AJ, Soriano A, Sánchez F, Correcher C, Orero A, et al. Design and evaluation of the MAMMI dedicated breast PET. Med Phys. 2012;39:5393.

[67] González-Montoro A, Sánchez F, Bruyndonckx P, Cañizares G, Benlloch JM, González AJ. Novel method to measure the intrinsic spatial resolution in PET detectors based on monolithic crystals. NIM(A) 2019;920:58-67.

[68] Lamprou E, Gonzalez AJ, Sanchez F, Benlloch JM. Exploring TOF capabilities of PET detector blocks based on large monolithic crystals and analog SiPMs. Physica Med 2020;70:10-8.

[69] Moehrs S, Guerra AD, Herbert DJ, Mandelkern MA. A detector head design for small-animal PET with silicon photomultipliers (SiPM). Phys Med Biol. 2006;51: $1113-27$.

[70] Kwon SI, Roncali E, Gola A, Paternoster G, Piemonte C, Cherry SR. Dual-ended readout of bismuth germanate to improve timing resolution in time-of-flight PET. Phys Med Biol 2019;64:105007.

[71] Kratochwil N, Gundacker S, Lecoq P, Auffray E. Pushing Cherenkov PET with BGO via coincidence time resolution classification and correction. Phys Med Biol 2020; 65:115004. 